\title{
A seropositive nodular rheumatoid polyarthritis without arthritis, does it exist?
}

\author{
Ben Fredj Ismail Fatma, Rezgui Amel, Karmani Monia, Ben Abdallah Olfa, Azzebi Samira, \\ Laouani Kechrid Chedia \\ Department of Internal Medicine, Sahloul Hospital, Sousse, Tunisia \\ Email: bfi.fatma@yahoo.fr
}

Received 14 January 2013; revised 15 February 2013; accepted 23 February 2013

Copyright (c) 2013 Ben Fredj Ismail Fatma et al. This is an open access article distributed under the Creative Commons Attribution License, which permits unrestricted use, distribution, and reproduction in any medium, provided the original work is properly cited.

\begin{abstract}
Introduction: The rheumatoid polyarthritis is the most frequent chronic polyarthritis. It affects essentially woman between 40 and 60 years old. Rheumatic subcutaneous nodules and tenosynovitis are usually associated with seropositive symptomatic rheumatoid polyarthritis. However, it is rare that they constitute the essential clinical expression of the disease. Case report: A 60-year-old woman was hospitalized for tumefaction of the dorsal face of the right hand evolved two months before. The clinical examination was found isolated subcutaneous nodules. The anatomopathological exam of one nodule ended in its rheumatoid origin and the MRI of a second one confirmed a tenosynovitis. Anti-CCP antibodies were positive. The patient has never suffered from arthralgias. The evolution was favourable under corticosteroid therapy, methotrexate and colchicine. Discussion: Rheumatic subcutaneous nodules and tenosynovitis are usually associated with seropositive symptomatic rheumatoid polyarthritis. It is rare that they constitute the essential clinical expression of the disease. Conclusion: The extra-articular appearances of the rheumatoid polyarthritis must not be underestimated especially since they can constitute-even rarely-the essential clinical expression of the disease.
\end{abstract}

Keywords: Rheumatoid Polyarthritis; Rheumatoid nodules; Seropositive; Tenosynovitis

\section{INTRODUCTION}

The rheumatoid polyarthritis (RP) is the most frequent shape of the chronic polyarthritis, affecting joints provided with a synovial membrane. It is characterized by often bilateral and symmetric arthritis of several articular groups touching preferentially wrists and hands, evolved by pushes towards the deformation and the destruction of affected joints articulations. It gets essentially women between 40 and 60 years. The rheumatic subcutaneous nodules and the tenosynovitis are usually associated with seropositive RP. However, it is rare that they constitute the essential clinical expression of the disease.

We report a new particular observation by its mode of revelation and its clinical evolution.

\section{CASE REPORT}

A 60-year-old woman to the histories of asthma under inhaled corticoids in the long term, consulted for tumefaction of the dorsal face of the right hand evolving for two months without triggering factor neither fever nor change of the general state. The interrogation found a notion of ancient arthralgia of big and small joints without arthritis and without any other systematic sign. The physical examination noted the presence of multiple mobile painful nodules on irregular surface of the dorsal face of the right hand as well as the front homolatéral arm (Figures 1 and 2); Making two for four centimeters of main line with pain in the articular mobilization without arthritis nor limitation of movements. Biologically, there was an inflammatory syndrome and negative rheumatoid factor. Besides, antibodies anti-cyclic citrullines peptides (antiCCP) were positive to $42 \mathrm{UI} / \mathrm{ml}$ and the antinuclear antibodies were negative. To the radiography of hands, we noted a demineralization in bands without osteolysis. An echography and a magnetic resonnance imaging of mild parts then a MRI found a tissular, vasculated and heterogeneous mass of four centimeters, of the right hand; evoking a tenosynovitis. This mass was associated with several other nodules and the biopsy the biggest revealed its rheumatoid nature in the anatomopathological examination. A treatment with colchicine, general and local corticosteroid therapy in infiltration and methotrexate was then established. The evolution at fifteen months 


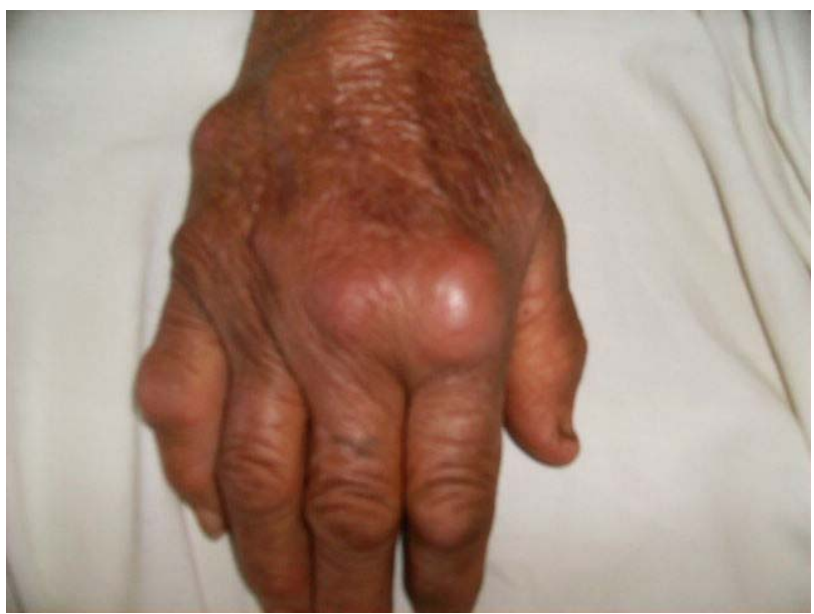

Figure 1. Multiple irregular nodules of the dorsal face of the right hand.

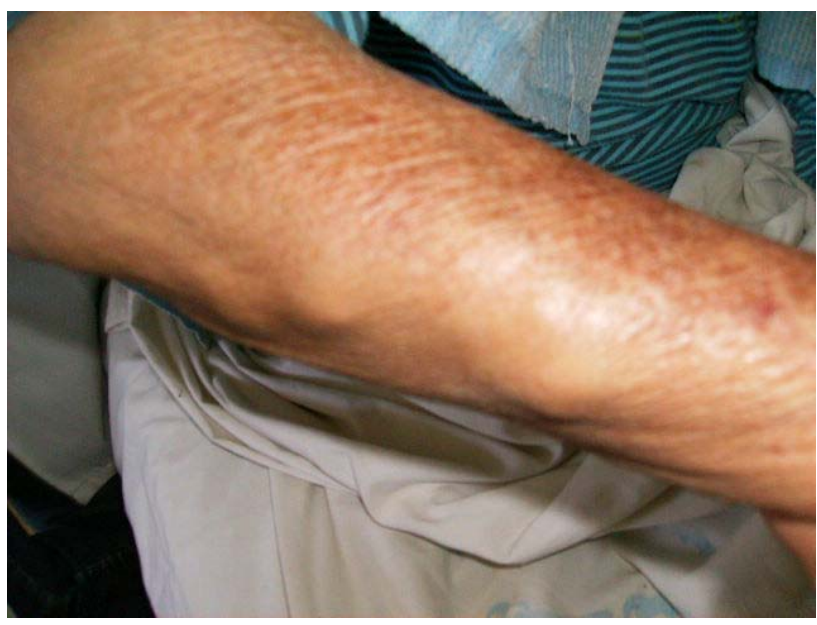

Figure 2. Multiple irregular nodules of the right arm.

was marked by a disappearance of rheumatoid nodules and the persistance of a little tumor corresponding to the tenosynovitis.

\section{DISCUSSION}

The rheumatoid polyarthritis (RP) is a chronic disease of complex and multifactorial determinism, unpredictable evolution and sometimes difficult positive and differential diagnosis. The ACR criteria of RP contain four articular clinical criteria on seven and remain debatable [1]. Rheumatoid nodules and factors give probably less to discussion [1]. The definition of the typical radiographic modifications of RP who constitutes the seventh criteria is probably very debatable. Besides, a radiographic reading made by a rheumatologist knowing the clinical symptomatologia influences the interpretation, in particular as regards the demineralization [1]. At our case report, the rheumatoid polyarthritis was essentially retained on the presence during the rheumatoid exploration of a nodule confirmed by the anatomopathology and the positive antiCCP antibodies. The sex and age (a 60-year-old woman), the tenosynovitis of the right hand, the notion of chronic arthralgia and the clinicobiologic evolution under treatment supported our diagnosis.

Classically, numerous arguments plead for an early diagnosis of RP. Effectively, osteocartilaginous destructions appear early in the evolution of the disease (10\% to $15 \%$ of the RP have erosions after three months of evolution, 30\% after one year, $70 \%$ after three years and 95\% after six years) and they are irreversible [2]. At our patient, the diagnosis of RP was able to be retained in spite of the absence of arthritis, thanks to the rather specific extra-articular appearances of the disease. The absence of true arthritis in our observation could be due to a low systematic passage of corticoids inhaled in the long term by the patient.

The rheumatoid factor is mostly absent during the first six months of evolution of RP. However, the presence of anti-CCP antibodies has a $50 \%$ of sensibility in a beginner RP, but especially a high specificity (97\%) [3]. It was the case in our observation.

A polyarthritis miming one PR can reveal other chronic inflammatory rheumatisms or even a neoplasm particularly a malignant lymphoma [4]. At our case report, the sex, the age, the interrogation, the clinical examination and the biological explorations eliminated other causes of arthralgia with subcutaneous nodules.

The imaging by magnetic resonnance can improve the diagnosis of tenosynovitis during RP [5]. In our observation it was in favour of a tenosynovitis and it eliminated the diagnosis of a malignant tumor and an infectious collection.

\section{CONCLUSION}

The extra-articular appearances of the rheumatoid polyarthritis must not be underestimated especially since they can constitute-even rarely - the essential clinical expression of the disease.

\section{REFERENCES}

[1] Saraux, A., Tobon, G., Jousse-Joulin, S. and DevauchellePensec, V. (2010) Les critères de classification et/ou de prédiction de la polyarthrite rhumatoïde. Revue du Rhumatisme, 77, 12-16.

[2] Carli, P., Landais, S., Aletti, M., Cournac, J.M., Poisnel, E. and Paris, J.F. (2009) Traitement actuel de la polyarthrite rhumatoïde. La Revue de Médecine Interne, 30, 10671079. doi:10.1016/j.revmed.2009.08.002

[3] Vallbracht, I., Rieber, J., Oppermann, M., Förger, F., Siebert, U. and Helmke, K. (2004) Diagnostic and clinical value of anti-cyclic citrullinated peptide antibodies compared with rheumatoid factor isotypes in rheumatoid 
arthritis. Annals of the Rheumatic Diseases, 63, 10791074. doi:10.1136/ard.2003.019877

[4] Gisserot, O., Leyral, G., El Baaj, M., Roméo, E., Bernard, P. and Carli, P. (2008) Une fausse polyarthrite rhumatoïde. La Revue de Médecine Interne, 29, 147-148. doi:10.1016/j.revmed.2007.05.017

[5] Chammas, M. (2005) Le poignet rhumatoïde. Annales de Chirurgie de la Main, 24, 275-298. doi:10.1016/j.main.2005.10.001 